\title{
KOMMENTAR
}

\section{Die Entdeckung einer Politik des Unpolitischen. Zur Institutionalisierung der „List der Vernunft“ in der Fiskalpolitik}

\author{
Roland Sturm
}

Charting the Unknown Territory of the Apolitical. The Institutionalization of "Rationality by Stealth" in Fiscal Policies

Abstract: In fiscal policy-making, though not exclusively here, we come across a paradox, namely the assumption that the best strategy for making political decisions is to move these decisions into the territory of the apolitical. Neutral rules and procedures are supposed to protect the essence of politics against party political competition. There are numerous examples which illustrate this. One could mention the "debt brake" or the EU fiscal pact. The conclusion of this comment is that the institutionalization of apolitical decision-making processes is only a detour which always leads right back into the heart of politics. There is no such thing as the politics of non-politics.

Keywords: fiscal policies, debt brake, government by experts, European Union, ESM

Schlagwörter: Fiskalpolitik, Schuldenbremse, Fiskalpakt, Expertenregierung, Europäische Union, ESM

\section{Funktionale Entpolitisierung?}

Die Finanzkrise mit ihren weitreichenden europäischen und nationalen Konsequenzen hat gezeigt, dass politische Entscheidungen nicht alleine mit Blick auf den durch Umfragen ermittelten Wählerwillen fallen (können). Wie ein Bankenrettungsplan im Detail ausgestaltet wird, oder welche institutionelle Form ein Euro-Rettungsschirm annimmt, interessiert das Wahlvolk nicht. Es nimmt an, dafür gebe es schon „Experten“. Mit dieser Meinung steht es nicht alleine. Auch politische Entscheidungsträger vertrauen auf den Rat von Anwaltskanzleien (Meßerschmidt 2012), IWF, Deutsche Bank oder orientieren sich an Rating Agenturen, die sich selbst als „impartial agency“ definieren (Mullard 2012). Dahinter steckt die Vermutung, es gebe eine Lösung, die gleichzeitig dem politischen Zweck dient und dem parteipolitischen Streit (zu Recht) entzogen sei. Das erinnert an die alte kommunalpolitische Weisheit, dass es keine „linke“ oder „rechte“ Straßenbeleuchtung gebe - wichtig sei alleine, ausreichend Licht.

Noch weiter in das Gefilde des Unpolitischen weisen jene politischen Weichenstellungen, die nicht nur auf einer eindimensionalen Lösungsvariante für ökono- 
mische Probleme bestehen (die TINA ${ }^{1}$-Variante), sondern einen Prozess initiieren, der eine gefundene „sachorientierte “ Lösung dauerhaft gegenüber dem Parteienwettbewerb abzuschotten sucht. Beispiele sind auf nationaler Ebene die „Schuldenbremse“ oder auf europäischer Ebene der „Fiskalpakt“. Hier misstraut die Politik grundsätzlich dem „unvernünftigen“ (nationalen) Parteienwettbewerb. Ja noch mehr - wie Alasdair Roberts argumentiert: Die Institutionalisierung von Fiskalregeln dokumentiere den Verlust des Vertrauens der politischen Elite in die Fähigkeit demokratischer Institutionen, wichtige Probleme eines Landes zu lösen (Roberts 2010).

Durch die Institutionalisierung „neutraler“ Entscheidungsregeln wird eine Paradoxie erzeugt: Was die Politik bei der Entscheidungsfindung entlasten soll, belastet sie gegenüber den Wählern. Diese haben mit Unterstützung der Parteien erfolgreich gelernt, dass bei gesellschaftlichen Problemen Responsivität und Transparenz von den Entscheidungsträgern eingefordert werden kann. In den Augen der Wählerinnen und Wähler existiert kein neutraler Sachzwang als Argument gegen ihre Interessen, und eigentlich ebenso wenig in den Augen der Politikerinnen und Politiker. Denn wie sonst ist zu erklären, dass diese bei der Aktivierung der mühsam im breiten Parteienkonsens gefundenen Regeln für politisch neutrales Entscheiden diesen Regeln häufig selbst kritisch gegenüber stehen, die Regeln breit interpretieren möchten oder so tun, als sei die Politik bei der Verabschiedung dieser Regeln nicht beteiligt gewesen?

Nicht nur „arguing“, sondern auch „bargaining“ ist aus wahltaktischen oder anderen Überlegungen (nationales Interesse, Schutz der einheimischen Wirtschaft) wieder erlaubt, blendet man die eigenen „stakes“ in dem Entstehungsprozess neutraler Regeln aus. Die wechselhafte Geschichte des europäischen Stabilitäts- und Wachstumspaktes demonstriert dies eindringlich gerade für die deutsche Position, die zunächst (1996) auf einer starren 3\%-Regel für die jährliche Neuverschuldung pochte, 2005 die Rationalität eines solchen „Brüsseler (!) Diktats“ in Frage stellte, und 2010 die Regeltreue zu einer unabdingbaren Voraussetzung finanzieller Seriosität in der Finanzkrise machte.

Eine besondere Form der Entpolitisierung von Politik ist die „Expertenregierung " bzw. die Herrschaft von Technokraten, wie sie jüngst in Griechenland und Italien erprobt worden ist. Hier konstituiert die Wahrnehmung europäischer Verpflichtungen, vor allem aber eine gemeinsame (nicht zuletzt vom Finanz- und Investmentunternehmen Goldman Sachs geprägte) Sicht auf ökonomische Zusammenhänge, eine angeblich wertfreie, nur dem wirtschaftlichen Funktionieren und der Überwindung endemischer Korruption verpflichtete Entscheiderelite. Ihre Legitimation bezieht sie nicht nur aus einem Wissensvorsprung gegenüber den Parteipolitikern und von außen durch die Anerkennung der europäischen Institutionen sowie zumindest teilweise der Retterkoalition des Euro (Troika), sondern gerade auch aus der Abkoppelung der Politik vom Parteienwettbewerb. Weil der Parteienwettbewerb aber für die Dauer einer Expertenregierung nicht suspendiert werden kann und da die politische Klasse der von Expertenregierungen gemanag-

1 There is no alternative - Margaret Thatchers Apodiktik, in deutscher Politikersprache: „alternativlos". 
ten Länder weder bereit war, die Schmach der zugeschriebenen Unfähigkeit noch den Verlust politischer Ämter zu akzeptieren, mobilisierte sie permanent gegen die Politik der Technokratenregierungen. Sie wurden - trotz kurzer Amtszeit - für die Strukturprobleme des Landes verantwortlich gemacht; der politische Normalbetrieb gab ihnen nicht die Zeit, die sie benötigt hätten, damit die Wirkung ihrer Maßnahmen hätten sichtbar werden können, und die politischen Parteien versuchten erfolgreich, die Legitimation der Expertenregierungen, die ihnen durch Anerkennung von Seiten europäischer Institutionen zuwuchs, umzudeuten in eine neue Form der (am populärsten: deutschen) Fremdherrschaft. Eigene Fehler der politischen Klasse wurden so schnell aus dem öffentlichen Bewusstsein verdrängt, und die Wiederherstellung „nationaler Selbstbestimmung“ durch die Vertreibung der Technokraten wurde zu einem nationalen Projekt gemacht, das allerdings in erster Linie dazu diente, den unreformierten alten Politikbetrieb wieder herzustellen. Erneut galt: Neutralität, die die Politik bei der Durchsetzung unpopulärer Entscheidungen entlasten sollte, führte zu mehr und nicht zu weniger „dysfunktionaler Politisierung “.

\section{Politikwissenschaftliche Zugänge zum Problem des Unpolitischen}

Die Politikwissenschaft hat bisher wenig Interesse an dieser Paradoxie des unpolitischen Regierens gezeigt. Für sie ist die Entkoppelung der Politik vom Parteienwettbewerb eher ein marginales Problem bzw. ein Problem, das gar nicht als Paradoxie wahrgenommen wurde. Wahrgenommen wurde die Trennung von Entscheidungsrationalität und kompetitiver politischer Präferenzbildung vor allem als ein anderer Modus von „Governance“ oder der „politischen Steuerung“. Nur - wer steuert bei Regeln und Prozessen, die deshalb erfunden wurden, weil „Governance“ und „politischer Steuerung“ regelmäßiges Versagen unterstellt wird? Wolfgang Streeck glaubt zumindest bei der Schuldenbremse die entscheidende „unsichtbare Hand“ gefunden zu haben: Die Märkte. Ihr Hauptziel, so Streeck, „muss sein, für den Krisenfall die Vorrangigkeit ihrer Ansprüche gegenüber denen des Staatsvolks - der Schuldenbedienung gegenüber der Daseinsvorsorge - zuverlässig zu sichern. Dies erreichen sie am besten durch im Idealfall verfassungsmäßig verankerte Institutionen wie die ,Schuldenbremse', die die Souveränität der Wähler und zukünftiger Regierungen über die öffentlichen Finanzen einschränken." (Streeck 2013, S. 126). Dies mag aus kapitalismuskritischer Sicht die Logik des Heilmittels erklären (obwohl man immer noch gerne wüsste, wer denn dann konkret die Interessen des „ideellen Gesamtkapitalisten“ umsetzt), nicht aber das Entstehen von Krisen und den Griff zur Politik des Unpolitischen.

In der Politikwissenschaft schien spätestens seit der Studie von James M. Buchanan und Richard E. Wagner (1977) klar, dass eine Politik, die den Bedarfsmeldungen des Wahlvolks folgt, um Politiker(innen) ihre Wiederwahl zu garantieren, den Staatshaushalt ruiniert. Public-Choice-Ansätze wie auch das AllmendeProblem weisen hier theoretisch in die gleiche Richtung: „Unter den gegebenen Bedingungen kann festgehalten werden“, so Ryczewski (2011, S. 118), „dass der einzelne Politiker sich im Hinblick auf die Wiederwahl rational verhält, wenn er neue Leistungen verspricht und durch Staatsverschuldung finanziert. Diese Praxis 
ist jedoch, gesamtwirtschaftlich betrachtet, irrational, weil sie nicht nachhaltig ist. " Dennoch wird eine solche Katastrophenstrategie verfolgt, denn der kurzfristige Wahlerfolg ist politisch ertragreicher als der langfristig gemeinwohlverträgliche Haushalt.

Wer rettet aber die Politik vor sich selbst? Hierzu wurde der deus-ex-machina der „unpolitischen Politik“ bzw. des neutralen Kontrollmechanismus erfunden, nicht nur in Deutschland und Europa: "Fiscal rules have come to be viewed as a replacement for the centrist coalitions, folkways, and even moral consensus that used to form the parameters and glue that sustained a responsible center for budgeting. A recent IMF article showed that 80 nations have fiscal rules compared to only 7 in 1990. Most have a combination of rules" (Posner u. Blöndal 2012, S. 22). Die Politik selbst setzt also auf die "List" der institutionalisierten Vernunft. Stanislaw Tillich (CDU), der sächsische Ministerpräsident, fand in einem Interview mit der FAZ (29.3.2011, S. 13) deutliche Worte: „Jemand muss die Politik disziplinieren. Maßstab sind Grundgesetz und Landesverfassung. Das Versprechen des Paradieses auf der Erde muss limitiert werden. "2 Gesucht wird also ein "Regelungskonzept, welches [im Idealfall, R. S.] die Verhaltensanreize der Akteure korrekt antizipiert, in die Schranken weist und so den für alle sonstigen Marktprozesse stets geforderten ,fairen Wettbewerb` auch für das Werben um Wählerstimmen verwirklicht“" (Ryczewski 2011, S. 118). Der Parteienwettbewerb wird unter dem Dach eines Regelwerkes domestiziert, soll aber dieses Regelwerk nicht in Frage stellen. Dies gelingt aber nur, wenn Politiker keine Anreize haben, aus dem Regelwerk auszubrechen (Hallerberg 2011).

War diese Zuflucht zu Fiskalregeln nötig? In der Literatur wird das zum Teil bestritten. In der Krise kann auch der „tugendhafte Politiker“ wieder entdeckt werden, der gerade die Sparpolitik zu einem Markenzeichen macht, um wiedergewählt zu werden. Im Vereinigten Königreich stehen alle Parlamentsparteien hinter dem Austerity-Projekt, was dort aus der Sparpolitik ein TINA-Modell macht. Empirisch konnten Paul Posner und Jón Blöndal (2012) zeigen, dass westliche Regierungen sogar schon vor der großen Krise nach 2007 vom Pfad des Wählerkaufs durch Ausgabenpolitik abwichen, ohne ihre Wiederwahl zu gefährden ${ }^{3}$. Ja selbst Einschnitte in das soziale Netz müssen kein elektoraler Selbstmord sein (Giger u. Nelson 2010). Dennoch glauben viele Politiker noch immer bzw. werden zu diesem Glauben durch die Budgetregeln der EU gezwungen, dass nur die Institutio-

2 Die gleiche Erwartung das Regieren verbessernder Folgen einer anthropomorph anmutenden Schuldenregel findet sich auch in der SPD. Deren damaliger rheinland-pfälzischer Finanzminister und deren damaliger Fraktionsvorsitzender im Deutschen Bundestag stellten fest: „Eine neue, kluge Schuldenregel sorgt besser vor und achtet auf die nachhaltige Entwicklung des Haushalts. [...] Eine Schuldenregel, die das leistet, hilft der Politik [sic!], notwendige Konsolidierungsschritte in die Wege zu leiten“ (Struck u. Deubel 2008, S. 8).

3 Die Autoren begründen dies mit der in der Politikwissenschaft verschwunden geglaubten programmatischen Ausrichtung der Politik, insbesondere in polarisierten Parteiensystemen, unabhängig von den Präferenzen des „median voter“ und der Fähigkeit der Politik, u. a. inkrementellen Wandel herbeizuführen, die Lasten „gerecht“ zu verteilen und die Vorteile der Sparpolitik zu kommunizieren. Zu Strategien für unpopuläre Haushaltsentscheidungen vgl. ausführlicher Wenzelburger 2011. 
nalisierung von „unpolitischen“ Budgetkontrollen und -regeln nationale Haushalte auf Dauer beherrschbar macht.

\section{Entpolitisierung in der politischen Praxis}

Zeigen lässt sich die Strategie des neutralen Kontrollmechanismus beispielsweise am Umgang des amerikanischen Kongresses mit dem US-Haushaltsverfahren und der seit 1917 bestehenden Schuldengrenze. Der amerikanische Kongress beschloss 1985, seinem eigenen Versagen beim politischen Management des Budgetprozesses durch mechanische Regeln für die Budgetfindung zu begegnen. Dem bestehenden Haushaltsprozess wurde mit dem Gramm-Rudman-Hollings Act (GRH) ein starrer Zeitplan mit Entscheidungszwängen übergestülpt. Die Missachtung dieser Zwänge führte zu automatischen Haushaltskürzungen (Sturm 1989, S. 198 ff.). Der Kongress reformierte zwar diesen Prozess im Detail, auch wegen eines Urteils des Supreme Courts, das die Berücksichtigung des Gewaltenteilungsprinzips anmahnte, bestätigte ihn aber mit dem Budget Enforcement Act von 1990 und dem Balanced Budget Act von 1997. Die neuen Regeln definierten wiederum Defizitgrenzen und verlangten eine Kompensation für neue Ausgaben durch höhere Steuern oder das Kürzen von Ausgaben. Als diese Regel aufgegeben wurde, womit der Zugang politischer Interessen zu den Haushaltsmitteln erleichtert wurde, entstanden erneut defizitfinanzierte Haushalte. Der wieder einsetzende politische Kampf von Demokraten und Republikanern führte die USA an den haushaltspolitischen Abgrund („,fiscal cliff“), der eigentlich gar kein Abgrund war, sondern nur Ausdruck des unlösbaren Konflikts zwischen zwei Interpretationen des Wählerwillens: mehr Ausgaben bzw. mehr Steuern (Demokraten) bzw. weniger Ausgaben und weniger Steuern (Republikaner). Hier erzeugte erst der Konflikt, der Mehrheitsentscheidungen im Kongress verhindert, eine imaginäre Defizitschranke, denn die Verschuldungsgrenze war hier zuvor immer entsprechend des haushaltspolitischen Bedarfs angehoben worden.

Politikwissenschaftler könnten sich also bestätigt fühlen, dass es de facto „unpolitische Politik “ gar nicht gibt. Der Economist (14.5.2011, S. 54) zitierte Susan Irving vom Government Accountability Office, einer überparteilichen Einrichtung des Kongresses, mit der zutreffenden Beobachtung „no process change can force agreement where one does not exist“. Dagegen könnte allerdings eingewandt werden, dass die Annahme eines rein pragmatischen Umgangs mit „funktionaler Entpolitisierung " deren legitimatorische Potenziale, z. B. im Falle der Schuldenbremse und des Fiskalpakts, unterschätzt. „Funktionale Entpolitisierung“, insbesondere, wenn sie den Charakter eines Prozesses annimmt, erfüllt geradezu paradigmatisch die von Niklas Luhmann (1969) definierten Voraussetzungen einer Legitimation von Politik durch Verfahren. Der Prozess ist normorientiert, weil er in der Regel auf gesetzlichen Vorgaben beruht (beispielsweise einer Änderung des Grundgesetzes bei der Schuldenbremse). Er besitzt Autonomie (die Schuldenbremse wird vom Stabilitätsrat überwacht) und ist ausreichend komplex, um Konflikte zuzulassen. Die offene Frage ist, ob die Beteiligten (im Falle des Stabilitätsrates sind es auch die Betroffenen) bereit sind, schon aufgrund ihrer Beteiligung die hier gefundenen Ergebnisse zu akzeptieren. Auch von außen wird Kritik von denjeni- 
gen laut, die dem Haushaltsausgleich nichts abgewinnen können und wie Die Linke und die Gewerkschaften darauf setzten, dass ein kruder Neokeynesianismus, der jegliche Ausgabenpolitik für sozial und wirtschaftspolitisch sinnvoll erklärt, auch wenn die Staatsverschuldung weiter wächst, die bessere Alternative ist (Sturm 2011).

Eventuell, so könnte man vermuten, sind zusätzliche Legitimationsgewinne zu erzielen, wenn Regeln sine ira et studio heute für die Zukunft gefunden werden. Das Bundesverfassungsgericht in seinem Urteil von 1999 zum Maßstäbegesetz ein weiteres Beispiel für eine „unpolitische“ Vorgabe (hier für den Länderfinanzausgleich) - bemühte gar das Rawlsche Bild des "Schleiers des Nichtwissens“, um die Vorteile einer nicht aus dem politischen Tageskampf entstandenen Regelstruktur zu betonen. Diese sollte folgendermaßen ausgestaltet sein: „Das Gesetz gestaltet in seiner formellen Allgemeinheit rational-planmäßig die Zukunft, setzt eine gewisse Dauerhaftigkeit der Regel voraus, erstreckt ihre Anwendung auf eine unbestimmte Vielzahl künftiger Fälle, verwirklicht damit Distanz zu den Betroffenen, wendet die Aufmerksamkeit des regelnden Organs dem auch für die Zukunft verpflichtenden $\mathrm{Maß}$ zu und wahrt die Erstzuständigkeit des Gesetzgebers bei der Verfassungsinterpretation“ (BVerfG, 2 BvF 2/98). Mit dieser Vorgabe des Bundesverfassungsgerichts wurde bei allen an der Entscheidung über die Ausgestaltung des Finanzausgleichs Beteiligten deren Vertrauen in eine „unpolitische“ Konfliktlösung getestet. Wir wissen, wie der Test ausfiel. Die Reaktion war ein umfassendes Misstrauensvotum gegenüber politisch neutralen Regeln. Bund und Länder einigten sich darauf, die zur Verfügung stehende Finanzmasse nach einem zähen Verhandlungsprozess geringfügig nach neuen Regeln zu verteilen. Diese wurden dann - entgegen den Intentionen des Bundesverfassungsgerichts - in den „Adelsstand“ von „Maßstäben“ erhoben, die angeblich den oben zitierten Ansprüchen des Gerichts genügten.

Auch die Schuldenbremse wurde 2009 als Zukunftsprojekt konzipiert, das für den Bund 2016 und für die Länder 2020 erst Realität werden soll. Für die politikwissenschaftliche Forschung stellt sich hier die Frage, ob Politik zu einer solchen Selbstbindung in der Zukunft überhaupt in der Lage ist (d. h. nicht, dass sie sich nicht bemüht). Oder ob geänderte Voraussetzungen, politische Kräfteverhältnisse oder neues politisches Personal dazu führen, die gefundene Selbstbindung nicht auf sich beziehen. Wäre letzteres der Fall müsste man sich um milliardenschwere „unpolitische“ Regelwerke auf der EU-Ebene, wie die Rettungsschirme oder den Fiskalpakt, nicht nur ernsthafte Gedanken machen. Man stünde auch vor dem Scherbenhaufen der Euro-Rettung, die ja auf der Vereinbarung kurzfristiger Hilfe für langfristige Rückzahlung plus Strukturreformen beruht.

Eine Abkoppelung politischer Entscheidungen vom Parteienwettbewerb fällt in der deutschen Politik beim Thema Europa am leichtesten. Der „permissive Konsens" in der Europapolitik ist in der Fiskalpolitik zu einem Elitenkonsens geworden, der die politischen Exekutiven weitgehend vor dem nationalen politischen Konkurrenzkampf bewahrt und an der politischen Wahrnehmung der Bevölkerung vorbei geht. „Der Zweck des Ganzen, dessen Erreichung immer näher rückt“, so Streeck (2013, S. 164), „ist die Entpolitisierung der Wirtschaft bei gleichzeitiger Entdemokratisierung der Politik.“ Auch wenn man diese radikale 
These Streecks nicht teilt, bleibt doch die empirisch belegbare Feststellung, dass die „Entpolitisierung“ der Fiskalpolitik in dieser Legislaturperiode im Deutschen Bundestag deutliche Fortschritte gemacht hat. Die Opposition im Deutschen Bundestag, mit Ausnahme der Linken, stimmte bei Abstimmungen über die Gesetze zur Bewältigung der europäischen Staatsschuldenkrise regelmäßig und mit der Mehrheit der Abgeordneten der SPD und B90/ Die Grünen den Vorschlägen der Bundesregierung zu bzw. enthielt sich - im Falle der SPD - wegen unzureichender Information durch die Bundesregierung einmal der Stimme. Fiskalpolitische Entscheidungen fielen mit Zustimmungsraten von ca. 80 Prozent im Deutschen Bundestag. Dies provozierte den ehemaligen Chef-Volkswirt der EZB, Otmar Issing, zu der Frage: „Welches Demokratieverständnis steht hinter dem Versuch, über eine klammheimliche Ausdehnung der Transferunion eine fiskalische Vergemeinschaftung zu erreichen, die man sich auf dem Weg der Vertragsänderung aus naheliegenden Gründen nicht zutraut?“ (Issing 2012).

\section{Zustimmung der Oppositionsparteien im Deutschen Bundestag zu Gesetzen zur} Bewältigung der europäischen Staatsschuldenkrise (Regierungsmehrheit $=53,3 \%$ )

\begin{tabular}{|c|c|c|c|c|}
\hline & $\%$ Bundestag & $\%$ SPD & \% B 90/Grüne & \% Die Linke \\
\hline 7.5.2010: Griechenlandhilfe ${ }^{1}$ & 63,1 & 2,0 & 82,3 & 0 \\
\hline 21.5.2010: ESM $^{2}$ & 51,5 & 0 & 0 & 0 \\
\hline $\begin{array}{l}\text { 29.9.2011: Erweiterung } \\
\text { Euro-Rettungsschirm }\end{array}$ & 84,5 & 94,5 & 94,1 & 0 \\
\hline 26.10.2011: Stärkung EFSF & 81,2 & 86,9 & 91,1 & 0 \\
\hline $\begin{array}{l}\text { 27.2.2012: } \\
\text { Zweite Griechenlandhilfe }\end{array}$ & 80,1 & 86,3 & 92,6 & 0 \\
\hline 29.6.2012: ESM & 79,8 & 87,6 & 95,5 & 0 \\
\hline 29.6.2012: Fiskalpakt & 78,8 & 78,7 & 79,4 & 0 \\
\hline $\begin{array}{l}\text { 19.7.2012: Hilfspaket für } \\
\text { spanische Banken }\end{array}$ & 76,4 & 80,1 & 79,4 & 0 \\
\hline
\end{tabular}

Quelle: Eigene Berechnungen nach spiegel online. Anmerkungen: ${ }^{~}$ Währungsunion-Finanzstabilitäts-Gesetz

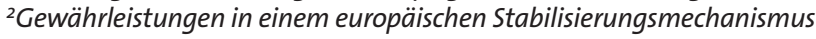

Aus deutscher Perspektive ist die EU für den nationalen Parteienwettbewerb schwer zugänglich. Die EU-Kommission ist ein frühes Modell eines Entscheidungsgremiums, das sich außerhalb von parteipolitischen und auch nationalen Interessen bewegen soll - nur den Verträgen und dem Gedanken der europäischen Integration verpflichtet. Die traditionellen Erklärungen für die Übertragung europäischer Präferenzen in nationales Recht bewegen sich im scheinbar unpolitischen Raum des Rechts (Phelan 2011). Dennoch setzen die Entscheidungen europäischer Institutionen den nationalen Parteienwettbewerb in Schwingung, nach Politikfeldern und Mitgliedstaaten in unterschiedlichem Maße. Die EU-Mitgliedschaft hat in einer Reihe von Ländern das Entstehen von anti-Europa Parteien provoziert, die in der EU selbst kein neutrales Gehäuse für die Verwirklichung nationaler Interessen sehen können. Die EU wird u. a. kritisiert als „Superstaat“ (Enzensberger 2011), der die nationalen Demokratien aushöhle, oder als „neoliberales 
Projekt", das den Sozialstaat abschaffen wolle (Streeck 2013). Aber auch bei EUBefürwortern bilden sich parteipolitisch unterschiedliche Präferenzen aus, z. B. was die Fiskalpolitik betrifft. Das Ausbleiben europapolitischer Kontroversen, das die deutsche Politik kennzeichnet, ist eher die Ausnahme. In anderen EU Mitgliedsländern, in denen eine pro-europäische Haltung weniger zum historischen Erbe gehört und einen verfassungspolitisch ${ }^{4}$ geringeren Rang hat, ist die Neigung parteipolitischer Polarisierung in der Fiskalpolitik weit größer.

\section{Lässt sich die deutsche Schuldenbremse entpolitisieren?}

Die Entpolitisierung der Schuldenbremse dürfte aus einer Reihe von Gründen schwer fallen. Die Politik hat sich Zugänge vorbehalten, die ihr Auswege aus vermuteten elektoralen Zwängen bieten. Am besten würde die Schuldenbremse funktionieren, wenn diese entpolitisierte Strategie politische Unterstützung fände. Wenn also alle Parteien zumindest bereit wären, nicht aus dem Korsett der Schuldenbremse auszubrechen, oder noch besser, wenn Wählerinnen und Wähler diejenigen Parteien bei Wahlen belohnen würden, die sich an Schuldenregeln halten (allerdings müssten diese in Deutschland erst einmal der breiten Öffentlichkeit ins Gedächtnis gerufen werden und auch mehr politische Unterstützung finden).

Zur parteiübergreifenden Anerkennung der Schuldenbremse in politisch heiklen Konstellationen genügt nicht, dass im Rechtsstaat das Recht potentieller Verbündeter der Politik bei der Durchsetzung von Regeln ist. Klagen vor dem Bundesverfassungsgericht nehmen Zeit in Anspruch. Über ihre fiskalpolitische Nachhaltigkeit wäre nachzudenken, zum Beispiel, wenn man an die Urteile des Bundesverfassungsgerichts oder des Landesverfassungsgerichts NordrheinWestfalens zu verfassungswidrigen Haushalten denkt. Diese die Politik tadelnden Urteile blieben für den Parteienwettbewerb weitgehend irrelevant. Es ist auch nicht auszuschließen, dass Gerichte ein Überdehnen der Regeln weiterhin als regelkonform interpretieren, weil sie ebenso politisch urteilen wie die politisch Handelnden, wenn auch nicht aus parteipolitischen Gründen. Ein gutes Beispiel hierfür ist das Urteil des Gerichts der Europäischen Union (EuGH) zum Rettungsschirm ESM, das sich die größte Mühe gibt, rechtlich neutrale Argumente für das Akkommodieren politischer Weichenstellungen zu finden (Adamski 2013).

Auch die Ökonomie ist ein unsicherer Unterstützer der Politik. Sie ist eine Sozialwissenschaft und keine Naturwissenschaft mit entsprechenden Gesetzmäßigkeiten, die zu einem objektiven Maßstab gemacht werden könnten. Das hält Ökonomen aber nicht davon ab, sich selbst als „neutrale“ Berater der Politik zu definieren. ${ }^{5}$ Bei der Schuldenbremse spielen Ökonomen eine Doppelrolle. Sie defi-

4 Vgl. GG. Artikel 23, Absatz 1.

5 Erinnert sei nur an die Bilanz des Sachverständigenrates zur Begutachtung der gesamtwirtschaftlichen Entwicklung auch schon zu ökonomisch ruhigeren und weniger globalisierten Zeiten (Hickel u. Mattfeldt 1983). Nikolaus Piper erzählt für Ökonomen mit Humor folgende Anekdote: „Ein Heißluftballon [...] ist vom Kurs abgekommen und treibt orientierungslos über Berge und Täler. Endlich sehen die beiden Piloten tief unten einen Wanderer., Wo sind wir?' rufen sie ihm zu. ,Ihr seid in einem Ballon!' lautet die Antwort. Worauf der eine Ballonfahrer zum anderen sagt: ,Die Antwort ist präzise, formal korrekt und absolut nutzlos. Der Mann muß ein Ökonom sein““ (Piper 1993). 
nieren einerseits, wann und zu welchem Umfang ein Schuldenabbau erforderlich ist, und andererseits eröffnen ihre Daten Wege zur Umgehung der Schuldenbremse. Bei beiden Rollen entstehen Ergebnisse, die parteipolitisch in weitem Maße interpretierbar sind.

Das wichtigste politische Einfallstor bei der Schuldenbremse ist die Unterscheidung zwischen struktureller und konjunktureller Verschuldung. Strukturelle Verschuldung soll für die Länder ausgeschlossen und für den Bund begrenzt werden (hier ist das volle Ausschöpfen des Rahmens von 0,35 Prozent des BIP zu erwarten, Ryczwewski 2011, S. 91), konjunkturelle Verschuldung ist möglich. Der Gesetzgeber geht von der Annahme aus, Volkswirte könnten exakt berechnen, welche Ausgaben sich konjunkturellen und welche sich strukturellen Zwecken zuordnen lassen - und zwar schon bevor das Haushaltsjahr beginnt. Die Konjunkturkomponente des Defizits soll sich ergeben als Produkt aus der Produktionslücke und der Budgetsensitivität. Unter Produktionslücke wird die Unter- oder Überauslastung der gesamtwirtschaftlichen Produktionskapazitäten verstanden. De facto handelt es sich hier um eine Schätzung, da der Bezugsrahmen das laufende Haushaltsjahr ist. Dem Gesetzgeber waren die mit diesen Regeln verbundenen Unsicherheiten wohl bewusst, denn er fordert, die entsprechenden volkswirtschaftlichen Verfahren in Übereinstimmung mit dem im Rahmen des Europäischen Stabilitäts- und Wachstumspakts angewandten Konjunkturbereinigungsverfahren regelmäßig zu überprüfen und fortzuentwickeln. Unter Budgetsensitivität wird ein Zahlenwert verstanden, der angibt, wie sich die Einnahmen und Ausgaben des Bundes bei einer Veränderung der gesamtwirtschaftlichen Aktivität entwickeln. Hier zeigt sich die problematische Annahme, man könne im Zeitalter der Globalisierung solche „neutralen“ Zahlenwerte für politische Entscheidungen zeitgerecht und exakt ermitteln oder gar im nationalen Rahmen finden (Sturm 2009, S. 496). Die OECD (2010, S. 69-70) stellt in diesem Zusammenhang bezeichnenderweise fest: „Im Rahmen der neuen Haushaltsregel ist die Ermittlung der Position der Wirtschaft im Konjunkturzyklus von zentraler Bedeutung. Es ist allerdings schwierig, ein verlässliches Maß für den Konjunkturzyklus zu ermitteln, da sich das Produktionspotenzial nicht beobachten lässt und jede Messung der Lücke zwischen effektiver und potenzieller Produktion somit in hohem Maße ungewiss ist und häufig Gegenstand erheblicher Revisionen im Zeitverlauf wird." Wo Unsicherheit über die Lage der Konjunktur besteht, gibt es optimistischere und pessimistischere Zahlenwerte - die Politik hat also die Wahl mehr oder weniger auszugeben. Hinzu kommt: Abweichungen aus konjunkturellen Gründen von der Nullverschuldungsregel, die auf einem Kontrollkonto verbucht werden, sind nicht einfach zurückzuzahlen, sondern konjunkturgerecht zurückzuführen, was erneut politisches Entscheiden erfordert.

Parteipolitische Definitionsmacht entsteht auch bei den Ausnahmen von der Schuldenregel. Dies sind vorgesehen im Falle von Naturkatastrophen oder außergewöhnlichen Notsituationen, die sich der staatlichen Kontrolle entziehen und die staatliche Finanzlage erheblich beeinträchtigen (Beispiele: Finanzkrise, deutsche Einheit). Gerade die Finanzkrise birgt beträchtliches Potential für eine Aussetzung der Schuldenregel. Gemessen an der „kreativen Überwindung“ der europäischen Verträge (z. B. die No-Bail-Out-Regel, Artikel 125 AEUV) sind die rechtlichen 
Hürden und die in Frage stehenden Summen im Falle der deutschen Schuldenbremse eher zu vernachlässigen. Gröteke und Mause (2009) argumentieren zudem, dass die deutsche Schuldenbremse schon alleine deshalb die Politik nicht wird zähmen können, weil es bei Fehlverhalten eines Landes/ des Bundes an der glaubwürdigen No-Bail-Out-Drohung fehlt. Bei der Schuldenbremse zeigt sich also: Was rechtlich schwach bis gar nicht sanktioniert ist und ökonomisch de facto kostenfrei ist, ist ungeeignet für die Absicherung einer parteipolitisch neutralen fiskalpolitischen Schranke.

\section{Gibt es erfolgreiche Wege zur Neutralisierung von Parteipolitik bei fiskalpolitischen Entscheidungen?}

Die Antwort ist nein. In Parteiendemokratien sind Strategien der Delegation von Verantwortung auf Regeln und Verfahren, sind Strategien des „blaming“ und „shaming“ letztendlich nur Umwege und Instrumente des Wettbewerbs um Wählerstimmen. Das muss man nicht bedauern. Politiker werden gewählt, um Verantwortung zu übernehmen. Der Gedanke, dass dieses Übernehmen von Verantwortung darin besteht, auf andere Personen oder juristische bzw. ökonomische Notwendigkeiten zu verweisen, ist nicht sonderlich sympathisch. Eben so wenig ist dies die Flucht aus der Verantwortung für das Budget durch die Suche nach „übergeordneten“ Vorgaben, deren Legitimation die politisch Entscheidenden nach Opportunität beurteilen. Schließlich geht es bei der Fiskalpolitik in Deutschland wie in Europa immer nur um die zwei gleichen einfachen Fragen: Wie hoch sollen die Einnahmen sein, und wie hoch sind die Ausgaben? Die Erkenntnis, dass Einnahmen und Ausnahmen zusammen hängen, ist keineswegs überraschend. Fritz Morstein Marx (1965) hat in der PVS schon vor fünfzig Jahren hierauf eindringlich hingewiesen und den Bezug zu einer verantwortungsvollen Haushaltspolitik hergestellt. Und Aaaron Wildavsky (1988) hat vor fast dreißig Jahren den offensichtlichen Zusammenhang von Budgetieren und Regieren mit folgender entwaffnender Fragestellung hergestellt: „If You Can't Budget, How Can You Govern?" Bis heute fehlt es - gerade der Fiskalpolitik - an Transparenz, einem langen Atem, Prinzipien- und Programmtreue und dem Bemühen um Gestaltung, statt des ad hoc-Reagierens und des geschmeidigen Regierens (Sturm 1992, S. 44). Vielleicht wäre die Parteipolitik eine weit geringere Herausforderung für das Gemeinwohl, wie dies wohl die Verfechter einer Politik des Unpolitischen befürchten, wenn es ihr gelänge, die schon lange und häufig gestellten Fragen zum Sinn und Zweck des Regierens, in parteipolitische Kalküle zu integrieren. 


\section{Literatur}

Adamski, Heiner. 2013. EuGH und ESM. Der Europäische Stabilitätsmechanismus ist europarechtskonform. Gesellschaft-Wirtschaft-Politik 62:119-127.

Buchanan, James M., und Richard E. Wagner 1977. Democracy in Deficit: The Political Legacy of Lord Keynes. Indianapolis: Liberty Fund.

Giger, Nathalie, und Moira Nelson. 2010. The electoral consequences of welfare retrenchment: Blame avoidance or credit claiming in the era of permanent austerity? European Journal of Political Research 50:1-23.

Gröteke, Friedrich, und Karsten Mause. 2009. Die deutsche „Schuldenbremse“: Ein wirksames Instrument zur Vermeidung eines Bailout? Zeitschrift für Wirtschaftspolitik 58:309-335.

Enzensberger, Markus. 2011. Sanftes Monster Brüssel. Der Spiegel 28.2.2011:108-111.

Hallerberg, Mark. 2011. Wirksame Abwehr gegen Schulden. Frankfurter Allgemeine Zeitung 4.2.2011:12.

Hickel, Rudolf, und Harald Mattfeldt (Hrsg.). 1983. Millionen Arbeitslose! Streitschrift gegen den Rat der Fünf Weisen. Eine Bilanz nach zwanzig Jahren. Reinbek: Rowolth.

Issing, Otmar. 2010. Die Währungsunion auf dem Weg zur Fiskalunion?. Frankfurter Allgemeine Zeitung 6.1.2010:10.

Luhmann, Niklas. 1969. Legitimation durch Verfahren. Frankfurt a. M.: suhrkamp.

Meßerschmidt, Klaus. 2012. Private Gesetzgebungshelfer - Gesetzgebungsoutsourcing als privatisiertes Regulierungsmanagement in der Kanzleiendemokratie? Der Staat 51:387415.

Morstein Marx, Fritz. 1965. Regierungsprogramm und Haushaltsplanung in vergleichender Sicht. Politische Vierteljabresschrift 6:442-464.

Mullard, Maurice. 2012. The Credit Rating Agencies and Their Contribution to the Financial Crisis. The Political Quarterly 83:77-95.

OECD. 2010. Deutschland. Paris: OECD.

Phelan, William. 2011. Why Do the EU Member States Accept the Supremacy of European Law? Explaining Supremacy as an Alternative to Bilateral Reciprocity. Journal of European Public Policy 18:766-777.

Piper, Nikolaus. 1993. Editorial. In Zeit der Ökonomen. Eine kritische Bilanz volkswirtschaftlichen Denkens, ZEIT-Punkte Nr. 3/1993, Hrsg. Theo Sommer, Hamburg: DIE ZEIT.

Posner, Paul, und Jón Blöndal. 2012. Democracies and Deficits: Prospects for Fiscal Responsibility in Democratic Nations. Governance. An International Journal of Policy, Administration and Institutions 25:11-34.

Roberts, Alasdair. 2010. The Logic of Discipline: Global Capitalism and the Architecture of Government. Oxford: Oxford University Press.

Ryczewski, Christoph. 2011. Die Schuldenbremse im Grundgesetz. Untersuchung zur nachhaltigen Begrenzung der Staatsverschuldung unter polit-ökonomischen und bundesstaatlichen Gesichtspunkten. Berlin: Duncker \& Humblot.

Streeck, Wolfgang. 2013. Gekaufte Zeit. Die vertagte Krise des demokratischen Kapitalismus. Berlin: Suhrkamp.

Struck, Peter, und Ingolf Deubel. 2008: Jetzt erst recht eine Schuldenregel. Frankfurter Allgemeine Zeitung 4.12.2008:8. 
Sturm, Roland. 1989. Haushaltspolitik in westlichen Demokratien. Ein Vergleich des haushaltspolitischen Entscheidungsprozesses in der Bundesrepublik Deutschland, Frankreich, Großbritannien, Kanada und den USA. Baden-Baden: Nomos.

Sturm, Roland. 1992. Regierungsprogramm und Haushaltsplanung in vergleichender Sicht. In Regieren in der Bundesrepublik 4. Finanz- und wirtschaftspolitische Bestimmungsfaktoren des Regierens im Bundesstaat - unter besonderer Berücksichtigung des deutschen Vereinigungsprozesses, Hrsg. Hans-Hermann Hartwich und Göttrik Wewer, 31-45. Opladen: Leske+Budrich.

Sturm, Roland. 2009. Föderalismusreform II: „Schuldenbremse“, neokeynesianischer Glaube an die Steuerbarkeit der Wirtschaft und das altbekannte Instrument der Politikverflechtung. Gesellschaft-Wirtschaft-Politik 58:487-499.

Sturm, Roland. 2011. Verfassungsrechtliche Schuldenbremsen im Föderalismus. Zeitschrift für Parlamentsfragen 42:648-662.

Wenzelburger, Georg. 2011. Political Strategies and Fiscal Retrenchment: Evidence from Four Countries. West European Politics 34:1151-1184.

Wildavsky, Aaaron. 1988. If You Can't Budget, How Can You Govern? In Thinking About America. The United States in the 1990s, Hrsg. Annelise Anderson und Dennis L. Bark, 266-275. Stanford: Stanford University Press.

\section{Autorenangaben:}

Prof. Dr. Roland Sturm,

Friedrich-Alexander-Universität Erlangen-Nürnberg, Institut für Politische Wissenschaft, Kochstr. 4, 91054 Erlangen, roland.sturm@fau.de 